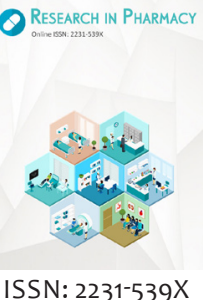

Received: December 03, 2020 Revised: February 08, 2021 Accepted: March 01, 2021 Published: March 11, 2021

*Corresponding Author: S. Gadge Sonali E-mail: sonaligadge94@ gmail.com

\section{Drug repurposing in the treatment of COVID-19: A review}

\author{
S. Gadge Sonalii, L. Salode Vikrant', D. Game Madhuri² \\ ${ }^{1}$ P. R. Patil Institute of Pharmacy, Talegaon (SP), Wardha, Maharashtra-442202, India, ${ }^{2}$ Vidyabharati College of \\ Pharmacy, C.K. Naidu Road, Amravati, Maharashtra-442202, India
}

\begin{abstract}
The first outbreak of severe acute respiratory syndrome coronavirus 2 (SARS-CoV-2) infectionwas in Wuhan, Hubei, China in December 2019 and declared as a pandemic by the World Health Organization. Currently, there is no proven effective vaccine or therapeutic agent to combat the deadly coronavirus disease. Currently, prevention from the infection, control measures and supportive care help to treat against COVID-19. Until an effective vaccine is available for COVID-19 infection, one can repurpose known therapeutic agents that block the entry of the virus into the host cell and control the COVID-19 infection. Drug repurposing is the new use of old drugs. In this review, the most common and possible drug treatment for COVID-19 is highlighted. The therapeutic agents include antiviral drugs like Remdesivir, chloroquine, hydroxychloroquine, lopinavir/ritonavir, umifenovir, favipiravir and oseltamivir and other agents. Clinical trials are ongoing to evaluate the safety and efficacy of these therapeutic agents in COVID-19 patients.
\end{abstract}

KEYWORDS: COVID-19, SARS-CoV-2, drug repurposing, remdesivir, chloroquine, hydroxychloroquine.

\section{INTRODUCTION}

World Health Organization declared coronavirus disease (COVID-19) outbreak as pandemic on March 12, 2020. The 2019 outbreak of Coronavirus Disease 2019 (COVID-19) was caused by a 2019-Novel coronavirus (2010-nCoV), later officially named as severe acute respiratory syndrome coronavirus 2 (SARS-CoV-2) by International Committee on Taxonomy of Viruses (ICTV). Like SARS-CoV and MERS-CoV, SARS-CoV-2 also belongs to the same family of Beta coronavirus (Xei and Chen, 2020). World Health Organization (WHO) declared COVID-19 as a Public Health Emergency of International Concern (PHEIC) on January 30, 2020; and a pandemic on March 11, 2020. The severity of this pandemic has led the world community to implement containment measures and to research collaboratively to address the knowledge gaps to identify COVID-19 therapies (Khuroo, 2020).

Coronaviruses $(\mathrm{CoV})$ consists of single-stranded RNA and these viruses can cause diseases to human and animals and are divided into four subfamilies (alpha, beta, gamma-, and delta$\mathrm{CoV}$ ), with SARS-CoV-2 belonging to the beta-CoV subfamily. The alpha and beta coronaviruses infect mammals, the gamma and delta coronaviruses infect bird. Historically, six $\mathrm{CoV}$ have been previously identified as human-infecting viruses: $\mathrm{HCoV}$ 229E, HCoV-0C43, SARS-CoV, HCoVNL63, HCoV-HKUl and MERS-CoV (Kantar et al., 2020).
SARS-CoV-2 got its name from the crown-like carbohydrate covered protein spikes sticking out from a relatively large viral centre with a diameter of $60-140 \mathrm{~nm}$. More than 1000 genomic sequences of COVID-19 have been globally reported in April 2020. 27 proteins have been identified within the SARS-CoV-2 genome; some are RNA-dependent RNA polymerase (RdRp). In addition, four structural proteins were shown to be encoded in the corona-viral genome: the nucleocapsid protein $(\mathrm{N})$, a small envelops protein $(\mathrm{E})$, the matrix proteins $(\mathrm{M})$, and the spike surface glycoprotein (S) (Zhu, et al., 2020; Zhau et al., 2020; Wu et al., 2020). The genetic code of COVID-19 was shown to be similar to that of SARS-CoV and MERS-CoV, but differences in protein structures.

No study proved due to the novel nature of the virus, which it will reduce men's fertility or sexual potency but medics in Wuhan have suggested the likelihood that the disease can affect the production of low sperm count and the formation of male sex hormones (low libido). Also, coronavirus attacks ACE2 receptors of the host cell which leads to COVID-19 related pneumonia, acute myocardial injury and chronic damage to the cardiovascular system (Zheng et al., 2020).

Currently, there is no vaccine or specific therapeutic agents for the treatment of COVID-19. Hence, it becomes a challenge to prevent and treat severely sick COVID-19 patients. Therefore, there is a requirement of effective vaccines are essential to combat against the deadly SARS-CoV-2. Presently, many

Copyright: (-) The authors. This article is open access and licensed under the terms of the Creative Commons Attribution License (http://creativecommons.org/licenses/by/4.0/) which permits unrestricted, use, distribution and reproduction in any medium, or format for any purpose, even commercially provided the work is properly cited. Attribution - You must give appropriate credit, provide a link to the license, and indicate if changes were made. 
research efforts have been devoted to developing vaccines all over the world. The drugs which are approved by the FDA in the USA for other indications have been used as "repurposed" drugs to treat COVID-19 patients, till we have specific therapeutic drugs or vaccines against SARS-CoV-2. This review will give an idea about the most current pharmacotherapeutics prescribed in the treatment of severe cases of COVID-19 patients. These include antiviral therapy, anti-inflammatory drugs (including anti-arthritis drugs), neuraminidase inhibitors, antibiotics, systemic corticosteroids and RNA synthesis inhibitors. Yet no specific antiviral drugs or vaccine is available for COVID-19. Hence, it is very essential to combat this deadly disease, which requires a clear understanding of disease pathology and critical strategies in identifying new drugs (Renyi et al., 2020).

Drug repurposing means examining existing drugs, they will find therapeutic effects on new diseases. Re-purposing existing drugs, or even rejected drugs are required to launch that increases the possibility of market success as well as reducing the costs and time (Aggarwal et al., 2020). The use of drug re-purposing is that drug details, such as the stages of chemical synthesis, mass production processes, various stages of clinical trials and many more have been identified earlier. Currently, there is no drug or vaccine for the prevention of the SARS-CoV-2 infection, but the use of available antivirals can be effective against this virus. For example, chloroquine and remdesivir (RDV, GS-5734) are two drugs that in vitro studies have suggested that they can inhibit the viral replication (Aanouz et al., 2020; Gupta et al., 2020).

\section{REPURPOSED DRUGS}

\section{Remdesivir}

Remdesivir is an antiviral agent, formally known as GS-5734 (Sanders et al., 2020) which was initially synthesized and developed by the Gilead Sciences for the treatment of Ebola virus infection in 2017 (Pawar, 2020). Remdesivir is considered as a potential drug for the treatment of COVID-19. It is a prodrug of molecular formula C27H35N6O8P and exact molecular mass $602.23 \mathrm{Da}$. In the body, it is metabolized into its active form known as GS-441524 with a molecular formula Cl2H13N5O4 and molecular mass 291.10 Da (Siegel et al., 2017). It is a monophosphamidate prodrug of an adenosine C-nucleoside having antiviral activity against several RNA viruses belonging to the family Coronaviridae (SARS-CoV, MERS-CoV), Paramyxoviridae (Nipah Virus and Hendra Virus) and Filoviridae (Ebola Virus; EBOV) (Amirian \& Levy, 2020). Remdesivir is now considering the promising potential therapy for COVID-19 due to its broad-spectrum antiviral activity.

Remdesivir as RNA-dependent RNA polymerase (RdRp) inhibitor, it inhibits the replication of multiple coronaviruses in respiratory epithelial cells. Once the remdesivir is added to the growing RNA chain (i position) it cannot stop immediate chain termination. The presence of the 3'-hydroxyl group continues the addition of three more nucleotides to stop the RNA synthesis at $(i+3)$ position. Thus, a possible mechanism of action is delayed RNA chain termination. In the recent studies, researchers found that almost identical results with SARS-CoV, MERS-CoV and SARS-CoV-2 RdRps. They found all the three coronavirus RdRp complexes terminate the RNA synthesis at $(i+3)$ position (Gordon et al., 2020).

Since all the viruses encode polymerases in the central steps of replication and transcription. Thus, polymerases are becoming the most attractive and suitable targets for the development of the antiviral activity. There are two known polymerase inhibitors: (i) Nucleoside and nucleotide substrate analogues and (ii) allosteric inhibitors. Nucleoside analogues are first triphosphated by the host cell to produce active inhibitors and then act as an inhibitor by competing with the natural nucleoside triphosphate and terminating the growing viral nucleic acids. Most of the antiviral drugs which are approved for the treatment of HIV therapy follow this mechanism (Uzunova et al., 2020).

Clinical trials are ongoing to evaluate the safety and antiviral activity of remdesivir in patients with mild to moderate or severe COVID-19 (NCT04292899, NCT04292730, NCT04257656, NCT04252664, and NCT04280705) (Sanders et al., 2020).

The use of remdesivir was successfully done in the USA for COVID-19 patient. The patient was treated with remdesivir on the evening of the 7th day of admission (Cao et al., 2020). The patient's clinical symptoms were improved after 2 days of the intravenous injection of the drug. It was also observed that their blood oxygen increased from $90 \%$ to $94 \%$. In a recent study, patients with COVID-19 received remdesivir treatment for 10 days, where on day 1, $200 \mathrm{mg}$ of the drug was administered intravenously and $100 \mathrm{mg}$ of the drug for 9 days. Clinical symptoms improvement and oxygen support observed in $68 \%$ of patients, of these $57 \%$ of patients were receiving mechanical ventilation and $13 \%$ of the patients died. Some adverse effects such as hypotension, rash, diarrhoea were observed in $57 \%$ of the patients who were receiving mechanical ventilation (Grein et al., 2020). Therefore, though remdesivir is a promising drug for the treatment of COVID-19, more clinical trials are required for the confirmation of its efficacy.

\section{Chloroquine and Hydroxychloroquine}

Quinine is obtained from the bark of cinchona trees which was first discovered in the 1600s and used as an antimalarial drug until the 1920s. During the Second World War, due to the inadequate amount of quinine under wartime pressure, synthetic drugs were derived from quinine. In 1934, German scientist Hans Andersag synthesized Chloroquine (CQ) as an anti-malarial drug. But, chloroquine was not used for medicinal purpose on a large scale due to its toxicity until it was re-synthesized by an American company and tested to be proved with efficacy and safety in early 1940. By introducing a hydroxyl group into $\mathrm{CQ}$, hydroxychloroquine (HCQ) was synthesized in 1946, and it was approved for medical use as an alternative to CQ because of its favourable efficacy and reduced toxicity in 1955. Since then, both CQ and HCQ have been used broadly for the prevention and treatment of malaria and in the 
treatment of chronic inflammatory diseases including systemic lupus erythematosus and rheumatoid arthritis (Browning, 2014; Lei et al., 2020).

The typical process of viral infection usually involves the following steps: endocytosis of viral particles; transport and uncoating leading to the release of the viral genome, transcription/translation/posttranslational modification of viral proteins and assembly followed byexocytosis. The infection process of COVID-19 has mediated through the interaction of spike (S) protein on virus and ACE2 on the host cell (Yan et al., 2020). CQ has been reported with the ability to inhibit glycosylation of the ACE2 receptor, which directly affects the spread of SARS-CoV infection in host cells (Vincent et al., 2005). Moreover, recently in silico simulation showed that $\mathrm{CQ} / \mathrm{HCQ}$ could prevent the access of $\mathrm{S}$ proteins to host cell surface ACE2 proteins by interacting with $\mathrm{S}$ proteins (Fantini et al., 2020). Therefore, inhibiting the interaction between $\mathrm{S}$ protein and ACE-2 might partially explain the prevention process. Additionally, CQ suppresses the expression of phosphatidylinositol binding clathrin assembly protein (PICALM), in addition, affects clathrin-mediated endocytosis of nanoparticles (Pelt et al., 2018). Coronavirus, after entry into the host cells, utilizes trypsin-like proteases in the lysosome to break the surface $\mathrm{S}$ proteins and promote the fusion with a lysosome in a $\mathrm{pH}$-dependent manner. Due to its basic properties, $\mathrm{CQ}$ accumulates in the acidic organelles such as lysosomes, that will lead to a change of their acidification status (Savarino et al., 2003). The elevated $\mathrm{pH}$ in lysosomes may inhibit the enzymatic activity of lysosome proteases and hence the infection process.

For the treatment of COVID-19, $500 \mathrm{mg}$ of chloroquine orally once or twice daily is recommended and for hydroxychloroquine dose of $400 \mathrm{mg}$ twice daily for 1 day followed by $200 \mathrm{mg}$ twice daily (Colson et al., 2020).

Chloroquine and hydroxychloroquine have been used worldwide for more than 75 years and listed as "Essential medicines" in the World Health Organization. It is cheap and established clinical safety profile (Cortegiani et al., 2020) but there is still a lack of evidence regarding the safety and effectiveness of these agents in treating SARS-CoV-2 (the novel virus causing COVID-19). In this regard, clinicians and patients should be made aware of the risk versus benefit profile of these medications (Juurlink, 2020).

\section{Lopinavir/Ritonavir}

Lopinavir/Ritonavir is a combined medication approved by the Food and Drug Administration which acts as a potent protease inhibitor for the treatment of HIV infection. It was identified in 1998 and approved in September 2000 (Yang et al., 2017). The combination was first marketed by Abbott under the brand name Kaletra in 2000. Lopinavir is available only in combination with Ritonavir to improve its bioavailability and biotransformation. Ritonavir is a potent inhibitor that helps in Lopinavir metabolism and improves its antiviral activity (Lv et al., 2015).
The protease is a homodimer containing 99 amino acids in each monomer and its active site contains three amino acids (Asp25-Thr26-Gly27) forms loop structure stabilizing the hydrogen bonds. The two aspartate residues (Asp 25 and Asp 250) from both chains are involved in drug binding. Lopinavir binds to the active site of the protease via a strong hydrogen bond with the oxygen of Asp 25 and the weak hydrogen bonds of the carboxylic acid of Asp 250. However, lopinavir rapidly metabolized in the liver by CYP3A4 and CYP3A5 and lowers its bioavailability. Ritonavir is found to inhibit CYP450-3A4 via its thiazole nitrogen and reduces its enzyme responsible for metabolizing drugs, such as antibiotics and protease inhibitors. Furthermore, the crystal structure of the complex CYP450-3A4/ ritonavir shows that the drug binds the active site of the enzyme by means of hydrophobic interactions. Therefore, ritonavir deactivates CYP450-3A4 and increases the bioavailability of certain drugs. Administration of ritonavir with lopinavir prohibits the metabolism of lopinavir by CYP450-3A4, which increases the lopinavir plasma concentration. Thus, it is used in the treatment of HIV infection. Lopinavir has antiviral effects against SARS-CoV-2. However, results from the treatment of patients with COVID-19 with lopinavir/ritonavir remain unclear (Kantar, 2020).

The dose treatment of lopinavir/ritonavir for the treatment of COVID-19 is $400 \mathrm{mg} / 100 \mathrm{mg}$ twice daily for 14 days plus standard of care. No benefits were observed with lopinavir/ritonavir apart from the standard care. The drug-drug interactions and adverse drug reactions are required to be monitored if this combined medication is used. More common adverse effects of lopinavir/ ritonavir are diarrhoea, nausea, abdominal pain, hepatotoxicity, dyslipidaemias. In patients with COVID-19, these adverse effects may be aggravated by combination therapy or viral infection because approximately $20 \%$ to $30 \%$ of patients have elevated transaminases at presentation with COVID-19. In a recent clinical trial, studies showed that around $50 \%$ of lopinavir/ ritonavir patients experienced adverse effects and around $14 \%$ of patients stopped therapy due to gastrointestinal adverse effects (Cao et al., 2020).

\section{Favipiravir}

Favipiravir (T-705 or 6-fluoro-3-hydroxy-2-pyrazinecarboxamide) is a synthetic pyrazinecarboxamide and an antiviral agent designed to treat influenza (Furuta et al., 2017). It shows broad- spectrum antiviral activity against $\mathrm{R}$ dependent RNA polymerase (Mentre et al., 2015). Favipiravir enters the infected cells through endocytosis and then transformed into favipiravir ribofuranosyl phosphate by phosphoriborobisylation and phosphorylation (Renyi et al., 2020). The antiviral activity is exhibited by targeting the RNA dependent RNA polymerase. Favipiravir has been used in the treatment of infectious disease caused by RNA viruses such as Ebola, influenza and norovirus. Clinical trials have been carried out for testing favipiravir in the treatment of COVID-19 in various countries like China and Japan. The clinical trial was performed in China in between February 202020 and March 122020 (Trial Registration Number: ChiCTR2000030254) [3] showed that treatment with favipiravir have recovery rate around $71.43 \%$ and duration of 
fever and cough relief time significantly shorter. However, more clinical trials are needed to be carried out to show whether favipiravir can have effective minimal antiviral activity against COVID-19. Some adverse effects experienced in the clinical trials that include higher concentration of uric acid in the serum with digestive tract complications (Chen et al., 2020).

\section{Umifenovir}

Umifenovir is also known as Arbidol. Chemically it is Ethyl6-bromo-4-[(dimethylamino) methyl]-5-hydroxy-1-methyl2[(phenylsulfanyl)-methyl]-1H-indole-3 carboxylate. It is an indole derivative. Umifenovir is an antiviral drug that was licensed in Russia in 1993 and in China in 2006 for the treatment of influenza A and B. Umifenovir also effective against $\mathrm{pH}$-dependent/independent viruses, such as a respiratory syncytial virus ( $\mathrm{Li}$ et al., 2018). Its mechanism of action is similar to Imatinib, an Abelson kinase inhibitor, the anchor drug in the treatment of Chronic Myeloid Leukemia. Both of these molecules inhibit the binding of the virus to the cell membrane. In January 2020, clinical trial conducted in Wuhan, China. 36 COVID-19 patients administered $400 \mathrm{mg}$ umifenovir three times a day for 9 days. In the control group, 31 untreated COVID-19 patients were selected. In this clinical trial, treatment with umifenovir showed a tendency to reduce viral load determined by RT-PCR and decreased mortality, as compared to the control group. Combination therapy of umifenovir and lopinavir/ritonavir was more effective than lopinavir/ritonavir only. Patients treated not only with protease inhibitor but also with umifenovir became sooner SARS-CoV-19 negative (Drozdzal et al., 2020). As reported by the clinical trial studies umifenovir might decrease the risk of SARS-CoV-19 and respiratory distress syndrome. Clinical trials with umifenovir alone or in combination with lopinavir/ritonavir have been recently initiated in China (Mckee et al., 2020).

\section{Oseltamivir}

Oseltamivir is approved by the Food and Drug Administration in 1999. It is sold under the brand name 'Tamiflu' and used in the treatment of influenza A and B. It is a neuraminidase inhibitor thatdistributed on the surface of the virus and inhibits the spread of influenza virus in the human body. There are no positive results were observed by the treatment of oseltamivir in COVID-19 patients. Clinical trials are still evaluating the efficacy of oseltamivir in treating SARS-CoV-2 infection. Oseltamivir in combination with chloroquine and favipiravir is also used in clinical trials (Jomah et al., 2020)

\section{Ribavirin}

Chemically ribavirin is 1-b-D-ribofuranosyl-1H-1,2,4-triazole3-carboxamide. It is a purine analogue which shows antiviral activity against DNA and RNA viruses. Ribavirin is used in the treatment of hepatitis $\mathrm{C}$ virus (Kantar et al., 2020). It is usually used in combination with interferon $\alpha$ (IFN) (Vellingiri et al., 2020). Currently, ribavirin is tested for the treatment of COVID-19, indicated that high concentrations of ribavirin required to cause significant reduction in the in vitro viral infection.

\section{Tocilizumab and Sarilumab}

Tocilizumab and sarilumab are monoclonal antibody that control cytokine release syndrome which is an inflammatory response caused by viral infections and certain drugs (Kim et al., 2015). Tocilizumab branded as Actembra that is humanized monoclonal antibodies developed by Roche and Chugai Pharmaceuticals for the treatment of rheumatoid arthritis. In China, it is expected to have a beneficial effect on coronavirus patient with severe lung damage and elevation in the interleukin 6 levels. In the open-label clinical study (ChiCTR2000029765) 21 patients with severe or critical COVID-19 infection treated with tocilizumab intravenously. The clinical-stage of 4 patients was classified as critical (19\%). All patients received standard of care including lopinavir and methylprednisolone, as well as tocilizumab at a dose of $400 \mathrm{mg}$ intravenously in one 4 or two doses. 18 patients received tocilizumab twice with the second dose being administered due to recurrent fever within 12 hours of first administration. After receiving tocilizumab, all patients experienced fever resolution with reported improvement of clinical symptoms. In $75 \%$ of patients, there was a significant increase in the oxygen level after receiving tocilizumab on the fifth day. No significant adverse reactions were reported during the clinical trial period (Kantar et al., 2020).

Sarilumab sold under the brand name'kefraza' that is humanized monoclonal antibodies developed by the Regeneron Pharmaceuticals and Sanofi for the treatment of rheumatoid arthritis. Sarilumab is an anti interleukin 6R antibody used in the treatment of rheumatoid arthritis. Regeneron Pharmaceuticals and Sanofi in the partnership planned a randomized clinical trial (NCT04315298) with the Northwell Health's Feinstein Institutes for Medical Research for March 2020 targeting to enroll 400 COVID-19 patients. Per cent change in C-protein and time to improve on a 7 -point scale based on death and type of hospitalization in patients with serum interleukin 6 levels above a threshold as primary endpoints (Renyi et al., 2020).

\section{Corticosteroids}

Corticosteroids are commonly used in a variety of inflammatory conditions. Additionally, they can be used in the form of pulse therapy to treat flares of autoimmune diseases. However, during the use of corticosteroids caution is needed due to the potential serious side effects associated with corticosteroid drugs and that corticosteroids generally suppress the immune system. The latter means that corticosteroids modulate hyper inflammation and, on the other hand, inhibit immune responses that are vital for the host defense against the virus (Saghazadeh et al., 2020).

Dexamethasone is an old corticosteroid. It is having broad antiinflammatory and immunosuppressant activity that reduces cell-mediated immunity and inhibits cytokine production. Its clinical indications spread from pain in the joints to asthma, irritable bowel disease/Crohn disease, emesis, multiple sclerosis 
and various autoimmune diseases, as well as different types of cancer, to name just the most prevalent. Dexamethasonehas been and is used to relieve the wheezing associated with COVID-19in many hospitals which were included in one arm of the Randomized Evaluation of COVid-19 thERapY (RECOVERY) trial [NCT04381936], an adaptive, multiple arms, randomized, controlled trial that in several months has enrolled more than 11,500 patients from over 175 National Health Service hospitals in the UK. Its immunosuppressant effect might benefit COVID-19 patients who experience CRS (Chakraborty et al., 2020).

\section{FUTURE PERSPECTIVE}

In addition to the drugs discussed in the review, many antiviral drugs have been explored for the treatment of COVID-19 for several months, without any positive effect. Other drugs tested for the treatment of MERS are nafamostat thymidine kinase inhibitors (ganciclovir and acyclovir), translation-inhibiting mRNA encapsulation inhibitor - ribavirin, nitazoxanide used to control helminthiasis and currently tested for viability in viral infections, another nucleotide analogue - penciclovir, as well as drugs known from HCV therapy (azvudine, danoprevir/ ritonavir, sofosbuvir/daclatasvir, and sofosbuvir/ledipasvir). No drugs which mentioned above are currently recommended for the treatment in SARS-CoV-2 infection. Due to the lack of availability of effective COVID-19 therapeutic agents, prevention of infection is essential. Additionally by isolating the sources of infection and hygienic measures, seems to be essential to focus on the development of a vaccine (Lythgoe \& Middleton, 2020; Li et al., 2020).

Many of the current drugs used for the treatment of COVID-19 patients include agents that are designed and available in the market for treating other viral infections, such as Malaria and EBOV. On the one hand, the use of repurposed drugs could be commercially applicable and a time-saving route; on the other hand, this suggests that these agents are not correct against COVID-19 and, hence, there is still a great deal of efficacy improvement through derivatization. The major problem with derivatization is the length of time required (at least 12-18 months) to design, synthesize, test, and acquire approval for any given new drug. As a result of the economic impact as well as the significant death rate of COVID-19, time is of the essence, and there is an immediate need to halt to interrupt the spread of this virus as soon as possible to avoid devastating global health and economic consequences. Thus, we must make do with currently available agents to contain the virus until treatment is on the market. Till the combination therapy using current agents helps to overcome the pandemic and should involve agents with significant enough activity against COVID-19 but with different mechanisms of action, because this will provide the best path towards over- coming viral resistance.

Another important aspect of this research for repurposed drugs involves patent protection issues under current national and/or international regulations. Therefore, the regulatory community must act to minimize any financial complications implicating private industry and update guidelines for drug licensure through repurposing if necessary. It should not escape us that this is a vital behind-the-scene act while efforts are underway to seek new indications for existing compounds (Novac et al., 2013).

The COVID-19 pandemic is unprecedented health, economic and humanitarian crisis that has a major and ongoing impact on people in every country around the world. It is also an example of the remarkable participation of scientists from every country united to find a cure and vaccine against one single virus, but also searching for future solutions (Lechowicz et al., 2020).

\section{CONCLUSION}

COVID-19 is a serious infectious disease has spread all over the world that is transmitted through physical contact. Coronavirus infects the host cell via spike protein and binds to the Angiotensin-Converting Enzyme 2 receptor. The most common symptoms of COVID-19 are fever, dry cough, tiredness and difficulty in breathing or shortness of breath. Researchers are continuously working for searching effective drugs in the treatment of COVID-19. Clinical trials are going for confirming the safety and efficacy of these repurposed drugs. Currently, there is no vaccine or specific treatment of antivirals for the treatment of COVID-19. In this review, the repurposed drugs mentioned having a different mechanism of action and act differently to combat COVID-19 infection. The repurposed drugs act by inhibiting viral infection by either binding to enzyme active sites and receptors or modulating intracellular signaling. No drug is having remarkable or unremarkable action against coronavirus but the use of the single drug may not be effective to control this deadly virus. So, the use of a combination of different antiviral agents with a different mechanism of action may be more effective but their adverse effects may not be underestimated.

\section{ACKNOWLEDGEMENT}

The authors are very thankful to the principal of P R Patil Institute of Pharmacy, Talegaon (SP) for valuable guidance.

\section{REFERENCES}

Aanouz, I., Belhassan, A., Khatabi, E. I., Lakhlifi, T., Idrissi, M. E., \& Bouachrine, M. (2020). Moroccan Medicinal Plants as inhibitors against SARS-CoV-2 main protease: Computational investigations. Journal of Biomolecular Structure and Dynamics. https://doi.org/10. 1080/07391102.2020.1758790

Aggarwal, M., Leser, G. P., \& Lamb, R. A. (2020). Repurposing papaverine as an antiviral agent against influenza viruses and paramyxoviruses. Journal of Virology, 94(6), 1-14. https://doi.org/10.1128/JVI.01888-19

Amirian, E. S., \& Levy, J. K. (2020). Current knowledge about the antivirals remdesivir (GS-5734) and GS-441524 as therapeutic options for coronaviruses. One Health. https://doi.org/10.1016/j. onehlt.2020.100128

Browning, D. J. (2014). Pharmacology of Chloroquine and Hydroxychloroquine. In Hydroxychloroquine and Chloroquine Retinopathy. New York, NY: Springer. https://doi.org/10.1007/9781-4939-0597-3_2

Cao, Y. C., Deng, Q., צ Dai, S. (2020). Remdesivir for severe acute respiratory syndrome coronavirus 2 causing COVID-19: An evaluation of the 
evidence. Trave/ Medicine and Infectious Disease, 35, 101647 https:// doi.org/10.1016/j.tmaid.2020.101647

Chakraborty, R., \& Parvez, S. (2020). COVID-19: An overview of the current pharmacological interventions, vaccines, and clinical trials. Biochemical Pharmacology, 180, 114184. https://doi.org/10.1016/j. bcp.2020.114184

Chen, C., Zhang, Y., Huang, J., Yin, P., Cheng, Z., Wu, J., Chen, S., Zhang, Y., Chen, B., Lu, M., Luo, Y., Zhang, J., \& Wang X. (2020). Favipiravir verses arbidal for COVID-19: a randomized clinical trial. MedRxiv. https:// doi.org/10.1101/2020.03.17.20037432

Colson, P., Rolain, J. M., Lagier, J. C., Brouqui, P., \& Raoult, D. (2020). Chloroquine and hydroxychloroquine as available weapons to find COVID-19. International Journal of Antimicrobial Agents, 55(4), 105932. https://doi.org/10.1016/j.ijantimicag.2020.105932

Cortegiani, A., Ingoglia, G., Ippolito, M., Giarrantano, A., \& Einav, S. (2020). A systematic review on the efficacy and safety of chloroquine for the treatment of COVID-19. Journal of Critical Care, 57, 279-283. https:// doi.org/10.1016/j.jcrc.2020.03.005

Drozdzal, S., Rosik, J., Lechowiz, K., Machaj, F., Kotfis, K., Ghavami, S., \& Los, M. J. (2020). FDA approved drugs with pharmacotherapeutic potential for SARS-CoV-2 (COVID-19) therapy. Drug Resistance Updates, 53, 100719. https://doi.org/10.1016/j.drup.2020.100719

Fantini, J., Scala, C. D., Chahinian, H., \& Yahi, N. (2020). Structural and molecular modeling studies reveal a new mechanism of action of chloroquine and hydroxychloroquine against SARS-CoV-2 infection. International Journal of Antimicrobial Agents, 55(5), 105960. https:// doi.org/10.1016/j.ijantimicag.2020.105960

Furuta, Y., Komeno, T., \& Nakamura, T. (2017). Favipiravir (T-705), a broad spectrum inhibitor of viral RNA polymerase. Proceedings of the Japan Academy, Series B Physical and Biological Sciences, 93(7), 449-463. https://doi.org/10.2183/pjab.93.027

Gordon, C. J., Tchesnokov, E. P., Feng, J. Y., Porter, D. P., \& Gotte, M. (2020). The antiviral compound remdesivir potently inhibits RNAdependent RNA polymerase from Middle East Respiratory Syndrome coronavirus. Journal of Biological Chemistry, 295(15), 4773-4779. https://doi.org/10.1074/jbc.AC120.013056

Grein, J., Ohmgari, N., Shin, D., Diaz, G., Asperges, E., Castagna, A., et al. (2020). Compassionate use of remdesivir for patients with severe COVID-19. The New England Journal of Medicine, 382(24), 23272336. https://doi.org/10.1056/NEJMoa2007016

Gupta, M. K., Vemula, S., Donde, R., Gouda, G., Behera, L., \& Vadde, R. (2020). In-silico approaches to detect inhibitors of the human severe acute respiratory syndrome coronavirus envelope protein ion channel. Journal of Biomolecular Structure and Dynamics. https://doi.org/10. 1080/07391102.2020.1751300

Jomah, S., Asdaq, S. M. B., \& Yamani, M. J. A. (2020). Clinical efficacy of antivirals against novel coronavirus (COVID-19) A review. Journal of Infection and Public Health. 2020; 13(9), 1187-1195. https://doi. org/10.1016/j.jiph.2020.07.013

Juurlink, D. N. (2020). Safety considerations with chloroquine, hydroxychloroquine and azithromycin in the management of SRASCoV-2 infection. Canadian Medical Association Journal, 192(17). 450-453. https://doi.org/10.1503/cmaj.200528

Kantar, S. E., Nehmeh, B., Saad, P., Mitri, G., Estephan, C., Mroueh, M., Akoury, E., \& Taleb, R. I. (2020). Derivatization and combination therapy of current COVID-19 therapeutic agents: a review of mechanistic pathways, adverse effects, and binding sites. Drug Discovery Today, 25(10), 1822-1838. https://doi.org/10.1016/j.drudis.2020.08.002

Khuroo, M. S. (2020). Chloroquine and Hydroxychloroquine in coronavirus disease 2019 (COVID-19). Facts, fiction and the hype: a critical appraisal. International Journal of Antimicrobial Agents, 56(3), 01-12. https://doi.org/10.1016/j.ijantimicag.2020.106101

Kim, G. W., Lee, N. R., Pi, R. H., Lim, Y. S., Lee, Y. M., Jeong, H. S., \& Chung, S. H. (2015). IL-6 inhibitors for the treatment of rheumatoid arthritis: Past, Present and future. Archives of Pharmacal Research, 38(5), 575-584. https://doi.org/10.1007/s12272-015-0569-8

Lechowicz, K., Drozdezal, S., Machaj, F., Rosik, J., Szostak, B., Baranska, M. Z., Biernawska, J., Dabrowski, W., Rotter, I., \& Kotfis, K. (2020). COVID-19: The potential treatment of pulmonary fibrosis associated with SARS-CoV-2 infection. Journal of Clinical Medicine, 9(6), 1917. https://doi.org/10.3390/jcm9061917

Lei, Z. N., Wu, Z. X., Dong, S., Yang, D. H., Zhang, L., Ke, Z., Zhou, C., \& Chen, Z. S. (2020). Chloroquine and Hydroxychloroquine in the treatment of malaria and repurposing in treating COVID-19.
Pharmacology and Therapeutics, 216, 107672. https://doi. org/10.1016/j.pharmthera.2020.107672

Li, H., Wang, Y. M., Xu, J. Y., \& Cao, B. (2020). Potential antiviral therapeutics for 2019 Novel Coronavirus. Chinese Journal of Tuberculosis and Respiratory Diseases, 43(0), 170-172. https://doi.org/10.3760/ cma.j.issn.1001-0939.2020.0002

Li, M. K., Liu, Y. Y., Wei, F., Shen, M. X., Zhong, Y., Li, S., Chen, L. J., Ma, N., Liu, B. Y., Mao, Y. D., Li, N., Hou, W., Xiong, H. R., \& Yang, Z. Q. (2018). Antiviral activity of arbidal hydrochloride against herpes simplex virus I in vitro and in vivo. International Journal of Antimicrobial Agents, 51(1), 98-106. https://doi.org/10.1016/j.ijantimicag.2017.09.001

Lv, Z., Chu, Y., \& Wang, Y. (2015). HIV Protease inhibitors: a review of molecular selectivity and toxicity. HIVIAIDS-Research and Palliative Care, 7, 95-104. https://doi.org/10.2147/HIV.S79956

Lythgoe, M. P., \& Middleton, P. (2020). Ongoing clinical trials for the management of COVID-19 pandemic. Trends Pharmacological Sciences, 41(6), 363-382. https://doi.org/10.1016/j.tips.2020.03.006

McKee, D. L., Sternberg, A., Stange, U., Laufer, S., \& Naujokat, C. (2020) Candidate drugs against SARS-CoV-2 and COVID-19. Pharmacological Research, 157, 104859. https://doi.org/10.1016/j.phrs.2020.104859

Mentre, F. Taburet, A. M., Guedj, J., Anglaret, X., Keita, S., Lamballerie, X. D. \& Malvy, D. (2015). Dose regimen of favipiravir for Ebola virus disease. The Lancet, infectious Diseases, 15(2), 150-151. https://doi. org/10.1016/S1473-3099(14)71047-3

Novac, N. (2013). Challenges and opportunities of drug repositioning. Trends in Pharmacological Sciences, 34(5), 267-272. https://doi. org/10.1016/j.tips.2013.03.004

Pawar, A. Y. (2020). Combating devastating COVID-19 by drug repurposing. International Journal of Antimicrobial Agents, 56(2), 01-04. https:// doi.org/10.1016/j.ijantimicag.2020.105984

Pelt, J., Busatto, S., Ferrari, M., Thompson, E. A., Mody, K., \& Wolfram, J. (2018). Chloroquine and nanoparticle drug delivery: A Promising combination. Pharmacology and Therapeutics, 191, 43-49. https:// doi.org/10.1016/j.pharmthera.2018.06.007

Renyi, W., Wang, L., Kuo, H. C. D., Ahmad, S. A., Peter, R., Chou, P. J., Li, S., Hudlikar, R., Liu, X., Liu, Z., Poiani, G. J., Amorosa, L., Brunetti, L., \& Kong, A. N. (2020). An update on current therapeutic drugs treating COVID-19. Current Pharmacology Reports, 6, 56-70 https://doi. org/10.1007/s40495-020-00216-7

Saghazadeh, A., \& Rezaei, N. (2020). Towards treatment planning of COVID-19: Rationale and hypothesis for the use of multiple immunosuppressive agents: Anti-antibodies, immunoglobulins, and corticosteroids. International Immunopharmacology, 84, 106560. https://doi.org/10.1016/j.intimp.2020.106560

Sanders, J. M., Marguerite, L. M., Jodlowski, T. Z., \& Cutrell, J. B. (2020) Pharmacologic Treatments for Coronavirus Disease 2019 (COVID-19) A review. Journal of American Medical Association, 323(18), 1824 1836. https://doi.org/10.1001/jama.2020.6019

Savarino, A., Boelaert, J. R., Cassone, A., Majori, G., \& Cauda, R. (2003) Effects of chlroquine on viral infections: An old drug against today's diseases. The Lancet, Infectious Diseases, 3(11), 722-727. https://doi. org/10.1016/s1473-3099(03)00806-5

Siegel, D., Hui, H. C., Doerffler, E., Clarke, M. O., Chun, K., Zhang, L. Neville, S., Carra, E., \& Solovena, V. (2017). Discovery and synthesis of a phosphamidate prodrug of a pyrrolol [2, 1-f][triazin-4-amino] adenine C-nucleoside (GS-5734) for the treatment of Ebola and emerging viruses. Journal of Medicinal Chemistry, 60(5), 1648-1661. https:// doi.org/10.1021/acs.jmedchem.6b01594

Uzunova, K., Filipova, E., Pavlova, V., \& Vekov, T. (2020). Insights into antiviral mechanisms of remdesivir, lopinavir/ritonavir and chloroquine/ hydroxychloroquine affecting the new SARS-CoV-2. Biomedicine and Pharmacotherapy, 131, 110668 https://doi.org/10.1016/j. biopha.2020.110668

Vellingiri, B., Jayaramayya, K., Iyer, M., Narayanasamy, A., Govindasamy, V., Giridharan, B., Ganesan, S., Venugopal, A., Venkatesan, D., Ganesan, H., Rajagopalan, K., Rahman, P., Cho, S. G., Kumar, N. S., \& Subramaniam, M. D. (2020). COVID-19: A promising cure for the global panic. The Science of the Total Environment, 725, 138277. https://doi.org/10.1016/j.scitotenv.2020.138277

Vincent, M. J., Bergeron, E., Benjannet, S., Erickson, B. R., Rollin, P. E., Ksiazek, T. G., Seidah, N. G., \& Nichol, S. T. (2005). Chloroquine is a potent inhibitor of SARS coronavirus infection and spread. Virology Journal, 2, 69. https://doi.org/10.1186/1743-422X-2-69

Wu, A., Peng, Y., Huang, B., Ding, X., Wang, X., Niu, P., Meng, J., Zhu, Z., 
Zhang, Z., Wang, J., Sheng, J., Quan, L., Zanzian, X., Tan, W., Cheng, G., \& Jiang T. (2020). Genome composition and divergence of the novel coronavirus (2019-nCoV) originating in China. Cell Host and Microbe, 27(3), 325-328. https://doi.org/10.1016/j.chom.2020.02.001

Wu, R., Wang, L., Kuo, H. D., Shannar, A., Peter, R., Chou, P. J., Li, S., Hudlikar, R., Liu, X., Liu, Z., Poiani, G. J., Amorosa, L., Brunetti, L., \& Kong, A. N. (2020). An Update on Current Therapeutic Drugs Treating COVID-19. Current Pharmacology Reports, 1-15. Advance online publication. https://doi.org/10.1007/s40495-020-00216-7

Xie, M., \& Chen, Q. (2020). Insight into 2019 novel coronavirus- an updated interim review and lessons from SARS-CoV and MERS-CoV. International Journal of Infectious Diseases, 94,119-124. https://doi. org/10.1016/j.ijid.2020.03.071

Yan, R., Zhang, Y., Li, Y., Xia, L., Guo, Y., \& Zhou, Q. (2020). Structural basis for the recognition of SARS-CoV-2 by full length ACE2. Science, 367(6485), 1444-1448. https://doi.org/10.1126/science.abb2762
Yang, W., Xiao, Q., Wang, D., Yao, C., \& Yang, J. (2017). Evaluation of pharmacokinetic interactions between long-acting HIV-1 infusion inhibitor albuvirtide and lopinavir/ritonavir, in HIV-infected subjects, combined with clinical study and simulation results. Xenobiotica, 47(2), 133-143. https://doi.org/10.3109/00498254.2016.1166532

Zhau, P., Yang, X. L., \& Zheng, L. S. (2020). A Pneumonia outbreak associated with a new coronavirus of probable bat origin. Nature, 579, 270-273. https://doi.org/10.1038/s41586-020-2012-7

Zheng, Y., Ma, Y.T., Zhang, J.Y., \& Xie, X. (2020). COVID-19 and the cardiovascular system. Nature Reviews Cardiology, 17, 259-260. https://doi.org/10.1038/s41569-020-0360-5

Zhu, N., Zhang, D., Wang, W., Li, X., Yang, B., Song, J., Zhao, X., Huang, B., Shi, W., Lu, R., \& Niu, P. (2020). A Novel coronavirus from patients with pneumonia in China, 2019. The New England Journal of Medicine, 382(8), 727-733. https://doi.org/10.1056/NEJMoa2001017 